\title{
Hand Schuller Christian Disease in Thoracic Spine: A Case Report and Literature Review
}

\section{Changqin Jiang | Hai Liu | Cong Dai | Wanwei Zhang | Wei Fang | Qiang Feng*}

*Correspondence: Qiang Feng,

Address: Radiology department, Yidu Central Hospital of Weifang Medical University, China

e-mail $\bowtie$ : fengqiang07@163.com

Received: 15 February 2021; Accepted: 18 February 2021

Copyright: (C) 2021 Jiang C. This is an open-access article distributed under the terms of the Creative Commons Attribution License, which permits unrestricted use, distribution, and reproduction in any medium, provided that the original work is properly cited.

\section{ABSTRACT}

Objective: The CT and MRI manifestations and clinical conditions of a thoracic HSC syndrome were analyzed. Plain CT imaging and reconstructions showed that T8 vertebral body was normal in shape, and map-like osteolytic bone destruction was seen on the left side and the vertebral arch with clear boundary. Soft tissue window showed a small amount of soft tissue mass on the left side of the vertebra. MR showed abnormal signal in the T8 spreading to the left vertebral arch. The findings showed that it is hypointense in T1 sequence, hyperintense in T2 and the fat-pressed sequence. The signal of intervertebral disc was normal. MRI enhanced scanning showed that T8 and its appendages were enhanced uniformly, and a few soft tissue around it was also enhanced in the same way. The postoperative histological examination was consistent with the Langerhans cell hyperplasia HSC disease of bone.

Keywords: Spine, Langerhans Cell Histocytosis, Computer Tomography, X-Ray Computed, Magnetic Resonance Imaging

\section{Clinical Case}

The patient was a 20-year-old male who complained of back pain for 2 months and progressive exacerbation for 1 month. No other discomfort symptoms were presented. Physical examination manifested as left side tenderness $(++)$. The muscle strength of both limbs is not weakened, and the pain and warmth are not decreased. Laboratory examination of C-reactive protein (-), hemogram (-). The past history, personal history and family history are not special.

\section{Image Features}

CT of thoracic vertebrae showed that the thoracic vertebrae was normal in physiological curvature. T8 vertebrae was normal in shape and height. Lamellar osteolytic bone destruction was seen in vertebrae and left vertebral arch. The boundary of the lesion was clear as a whole, local bone cortex disappeared, and the abnormal soft tissue tumor was formed. No obvious abnormality was found in the intervertebral space (Fig. 1 CT). 
On the plain MRI scan of thoracic vertebrae, the lesions of T8 vertebrae and left arch showed the lesion with hypointense on T1WI, hyperintense on T2WI and T2 lipid suppression sequence, surrounded by soft tissue, no disc involvement. The other vertebrae showed no obvious abnormality in morphology and signal. No abnormality was found in the shape and signal of all intervertebral discs, and no obvious stenosis was found in the same layer of vertebral canal and intervertebral foramen. No abnormal signal shadow was found in the spinal cord (Fig. 2a-d). MRI enhancement of thoracic vertebrae showed that the abnormal signals in T8 vertebrae were enhanced significantly, soft tissue around the vertebrae was also enhanced uniformly.There was no abnormal enhancements in other vertebrae (Fig. 2e).

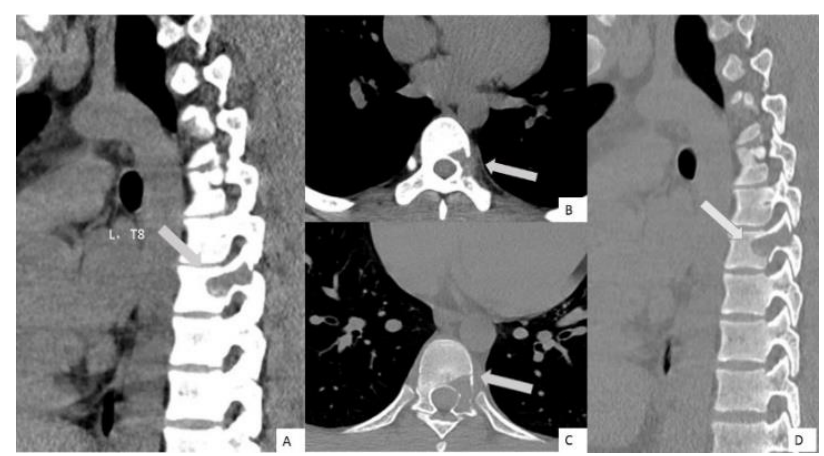

Figure 1: Plain CT scan of thoracic spine (A: sagittal reconstruction in soft tissue window; B: axial soft tissue window; C: axial bone window; D: sagittal reconstruction) showed osteolytic bone destruction with some soft tissue mass formed in the T8 vertibrea and the left arch.

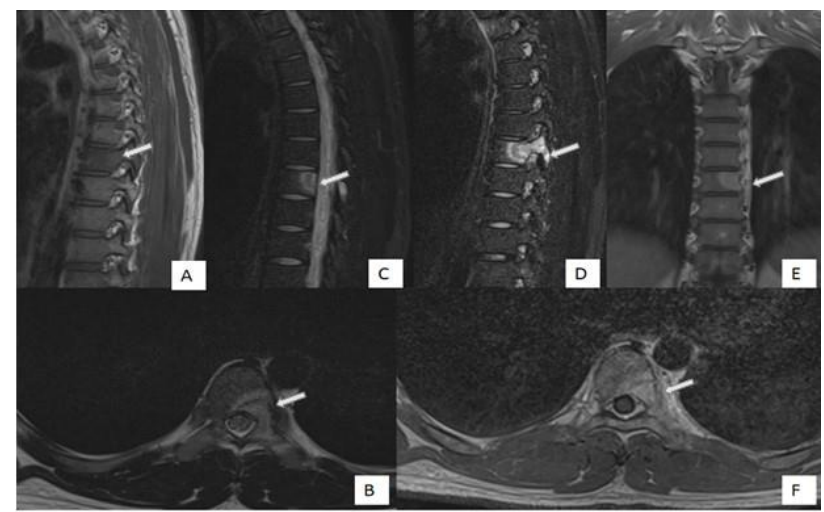

Figure 2: MR Plain and enhancement scan of thoracic spine. (A, T1WI sagittal plain scan; B, T2WI axial plain scan; C\&D: T2WI sagittal lipid sequence; E: T1 sequence coronal enhancement; F: T1 axial enhancement) T8 vertebral body and the left vertebral arch showed abnormal signal with hypointense in T1, hyperintense in T2 and fat-suppression sequence. Enhanced scanning showed obvious enhancement of the lesion uniformly.

\section{Histological Examination}

The patient underwent CT-guided biopsy examination, the pathological and immunohistochemistry results displayed as follows: CD1a (-), CD20 (-), CD21 (-), vimentin $20(+)$, CK (-), 
EMA (-), HLA-DR (+), Ki-67 $(20 \%+)$, langerin (+), lysozyme (+), S-100 (+). (Fig. 3). The histological results indicated a disease of Langhans Cell Hyperplasia of Hand Skull Christian syndrome (Arico et al., 2003).

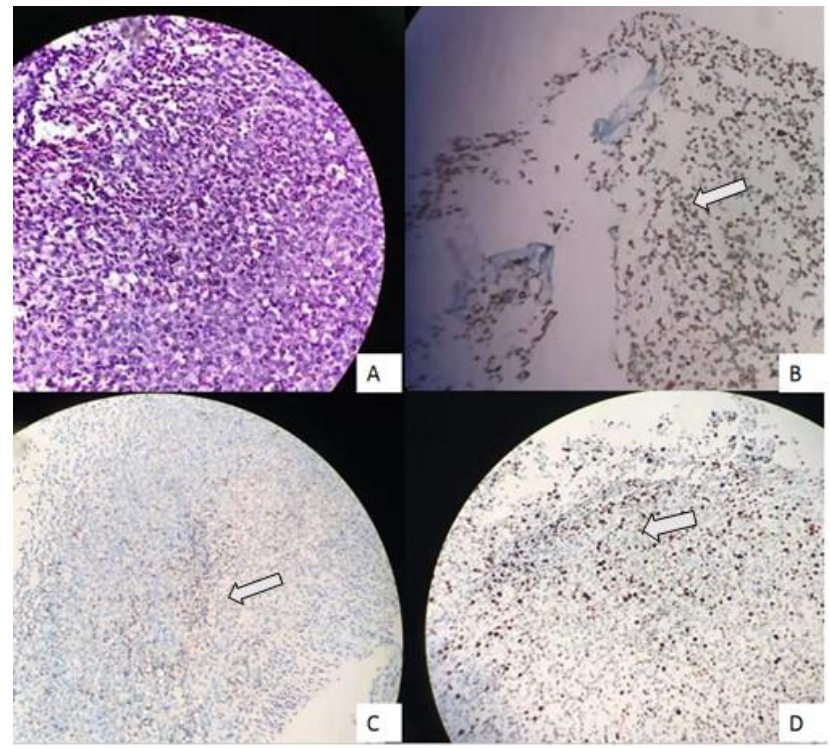

Figure 3: Pathology and partial immunohistochemistry. (a)HE $\times 40$; (b) vimentin 20; (c) S-100; (d) Ki-67 > 20\%. Under the microscope, extensive eosinophilic Langerhans cells and vimentin 20 and S-100 dyed positive were seen.

\section{Follow Up}

After the definite diagnosis of LCH, the patient did not take special treatment. He went back home and kept observing the disease, and the pain was relieved gradually. Three months later, the patient's pain symptoms were obviously relieved and he reexamined MRI scan, the image performance was shown in Fig. 4.

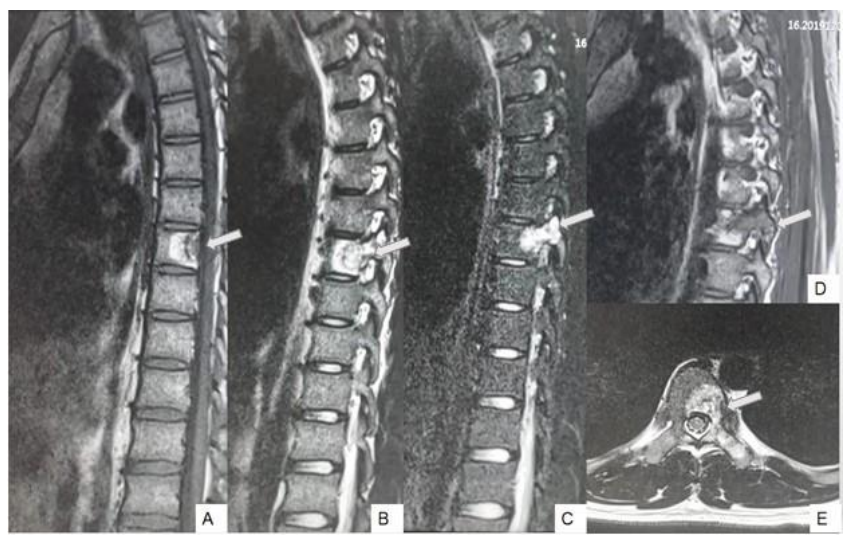

Figure 4: Plain MR scan (a, sagittal T1WI; B, sagittalT2WI; C, sagittal fat suppression T2; D, T1WI sagittal; E, axialT2WI) showed that theT8 vertebral body's shape is normal. Abnormal signal in the vertebral body and left vertebral arch and accessory was observed in T1WI sequence with hyperintense, mixed hyperintense in T2 pressure and fat suppression sequence. 


\section{Discussion}

In the WHO classification of bone tumors (Fourth Edition) in 2013, LCH was classified as "Tumors without definite tumor properties", which was positive to define LCH as an intermediate (locally invasive) tumor (Aricò et al., 2003). Hand Skull Christian disease belongs to the acute inflammatory phase of bone LCH, which is manifested with bone destruction, histiocytosis and eosinophilic cell infiltration. The other two manifestations are letterer Siwe syndrome and eosinophilic granuloma (Arico et al., 2003; Sweasey and Dauser, 1989). The disease usually occurs on teenagers and children, less in adults. The ratio of male to female is about $2.5: 1$. The most common incidences of the disease are skull, rib, pelvis, long bones of limbs, and rarely in spine (Sweasey and Dauser, 1989; Reddy et al., 2000; Lee et al., 2017; Lazio and Stambough, 1994).

In this case, the onset age is not common in adult male, no abnormality was found in the vertebral morphology on the image either. However, there are still some characteristic signs indicating it probably benign, such as clear sclerosis border, slit shadow in the center of the lesions. In addition, after 3 months of follow-up, the scope of the lesion is limited, the sclerotic edge (arc-like hypointense in the T2WI imaging of MRI) is clearer, and the shape of the vertebral body is still normal, which further suggests the lesions to be benign. Previous reports (Sweasey and Dauser, 1989; Reddy et al., 2000) have shown that LCH of spine is osteolytic destruction of vertebra in different degrees on CT, and residual small bone fragments and soft tissue shadows can be seen in or around some lesions, most of them are accompanied with slight bone expansion, and slight sclerosis can be seen at the edge. In the active stage, bone destruction is the main manifestation, while in the repair stage, osteolytic destruction reduced whereas bone hyperplasia and sclerosis increased (Reddy et al., 2000). Hence the density increased in the CT images. The vertebra is compressed and flattened to be wedge-shaped, and obviously flattened vertebra can be shown as "copper coin sign" (Sweasey and Dauser, 1989; Zhang et al., 2010). MRI mainly showed abnormal changes in the shape, indicating a structural changes in the abnormal signal in the diseased vertebra. With the development of the disease, the vertebra compressed and flattened or disc-shaped, while the intervertebral disc presented no obvious involvements (Zhang et al., 2010; Krengli et al., 1996). In this case, we noticed that in the MRI images follow-up after 3 months, T1WI showed flaky hyperintense and adverse signal in the lipid compression sequence, which indicating an increased proportion of fatty composition in the lesion area. No relevant reference had reported (Sweasey and Dauser, 1989; Zhang et al., 2010) However the related pathological reference (Zhang et al., 2010; Huang et al., 2013; Li Ruiqi, 2015) indicated that the mononuclear macrophages could engulf a lot of fat to form foam cells during the process of inflammation. So we speculate that this may be the oriented source of high signal on T1 sequence. 
The gold diagnosed standard of LCH is histological examination, that is, the characteristic of Langerin or Birbeck particles are found under the electron microscope (Sweasey and Dauser, 1989; Azouz et al., 2005). The pathological changes are composed of foam cells of mononuclear cells, eosinophils infiltrating and mononuclear macrophages that consume large amounts of fat (Sweasey and Dauser, 1989; Reddy et al., 2000). The disease could be divided into three stages through different pathological stages. First, proliferative stage, Langerhans cells gather and proliferate, the extensive infiltration causes osteoporosis. Second, granulomatous stage, with the progress of the disease, granuloma replaces the normal bone tissue, forming dead bone defects. Third, fibrotic repair stage, the disease is repaired, the granuloma will be replaced by fibrous tissue, and bone hyperplasia and sclerosis can be seen (Zhu et al., 2003). When the disease is in different stages of inflammation (Sweasey and Dauser, 1989; Reddy et al., 2000; Zhu et al., 2003), there will be completely different imaging manifestations. In the acute stage, LC cells proliferate in large numbers and soft tissue masses form. In the repair stage, the inflammatory components were absorbed, Langerhans cells were reduced, and the soft tissue masses were reduced or disappeared. During the long-term inflammatory and repair process of LCH (Lee et al., 2017), the trabecular structure of bone was destroyed and collapsed, forming the sign of "Tongqian vertebra".

Since age and course of spinal LCH are different, the imaging manifestation vary, and it has been mentioned in previous literature the the single lesion of vertebrea is the most common (Zhu et al., 2003). This case is single onset and involves the vertebral arch, which could be easily misdiagnosed clinically. In clinic, it should be differentiated from the following diseases. First, spinal tuberculosis, Mycobacterium tuberculosis firstly destroy the vertebral endplate, and then invade the intervertebral disc through the destruction channel, which is easy to invade the adjacent intervertebral disc and cause the adjacent intervertebral space to be narrow (Azouz et al., 2005; Zhu et al., 2003), which are the most important differentiation points between spinal tuberculosis and spinal LCH. Spinal tuberculosis is mostly osteolytic destruction of two adjacent vertebrae, most of the lesions are located in the back of the vertebrae, and the bone destruction is serious, which is fragmentary and wormlike, often accompanied by sand like dead bone, and the edge of the lesions is sclerotic (Zhang and Yuan, 2019). In addition, spinal tuberculosis is common to spread to the paravertebra and form a soft tissue abscess or to spread backward and form extradural abscess. MRI showed hypointense on T1WI and mixed hyperintense on T2WI both in the lesion and the intervertebral disc. Hypointense on T1WI and mixed hyperintense on T2WI when cold abscess was formed in soft tissue around the spine. In addition, most of the patients were complicated with tuberculosis poisoning symptoms, such as low fever, night sweat, and tuberculosis infection history. Second, pyogenic spondylitis, pyogenic inflammation is an acute nonspecific inflammatory response caused by non-specific bacterial infection. In the process of 
inflammatory response, a large number of neutrophils secrete proteolytic enzymes, which directly dissolve and destroy the intervertebral disc (Garg and Somvanshi, 2019). Pyogenic spondylitis can affect the vertebra and the intervertebral disc (Garg and Somvanshi, 2019). The osteosclerosis around the focus is obvious, paravertebral soft tissue abscess is diffuse, the boundary is unclear, and the vertebra basically maintains the original height. On T1WI, the signal intensity of the involved vertebra and the intervertebral disc increased, the volume of the intervertebral disc became smaller or disappeared, the residual part showed slightly lower or slightly higher signal, the fissure in the normal nucleus pulposus disappeared, and the sequence of lipid suppression showed equal high signal (Zhang and Yuan, 2019; Garg and Somvanshi, 2019) [12-13]. Most of them have the symptoms of persistent irritating, swelling and hot pain in clinic (Garg and Somvanshi, 2019). The leukocyte is significantly increased in laboratory examination, combined with clinical manifestations and laboratory examination, all those are helpful to distinguish them (Garg and Somvanshi, 2019). Third, spinal metastases, most of the metastases have a history of primary tumor. When several vertebral bodies are destroyed, and the lesions are distributed in a jumping pattern, often accompanied by pedicle destruction, no involvement of the intervertebral disc, and the paravertebral soft tissue masses are relatively limited (Xu Chen et al., 2015). MRI enhancement shows circumferential enhancement around the vertebral body, and most of the vertebral bodies are inhomogeneous and strong (Xu Chen et al., 2015). Its clinical symptoms are more severe and the the patients suffered obvious pain. So it is easy to distinguish them by comprehensive clinical data and imaging characteristics (Xu Chen et al., 2015).

At present, there are no unified treatment guidelines for spinal LCH. Symptomatic support treatment is mostly used, including observation, support protection, low-dose radiotherapy and chemotherapy or surgery (Li Haogang et al., 2012). However, there is no evidence so far that clinical intervention can effectively affect the natural course of spinal LCH (Li Haogang et al., 2012; Haupt et al., 2013), and cause radiotherapy can result in spinal endplate injury, hence it is not recommended to the clinic first, especially for young people (Jiang et al., 2011). Some lesions can be treated surgically when the adjacent central nervous system is compressed.

\section{Conclusions}

The spinal LCH is rare in adults, when adults have vertebral bone destruction, and the adjacent intervertebral space is normal, there is no obvious infection or tuberculosis poisoning symptoms or the history of primary tumor, the possibility of LCH should be considered. The follow up image manifestations of the recovery period in the natural course of thoracic LCH showed unique high signal on T1WI sequence compared with the previous image reports, combined with the clinical symptoms relieved, which enriches our understanding of the natural courses of the LCH. 


\section{References}

Arico M, Girschikofsky M, Généreau T, Klersy C, McClain K, Grois N, Emile JF, Lukina E, De Juli E, Danesino C. Langerhans cell histiocytosis in adults. Report from the International Registry of the Histiocyte Society. Eur J Cancer 2003; 39: 2341-2348.

Azouz EM, Saigal G, Rodriguez MM, Podda A. Langerhans' cell histiocytosis: pathology, imaging and treatment of skeletal involvement. Pediatr Radiol 2005; 35: 103-115.

Garg RK and Somvanshi DS. Spinal tuberculosis: a review. J Spinal Cord Med 2011; 34: 440-454.

Haupt R, Minkov M, Astigarraga I, Schäfer E, Nanduri V, Jubran R, Egeler RM, Janka G, Micic D, Rodriguez-Galindo C, Van Gool S. Langerhans cell histiocytosis (LCH): guidelines for diagnosis, clinical work-up, and treatment for patients till the age of 18 years. Pediatr Blood Cancer 2013; 60: 175-184.

Huang WD, Yang XH, Wu ZP, Huang Q, Xiao JR, Yang MS, Zhou ZH, Yan WJ, Song DW, Liu TL, Jia NY. Langerhans cell histiocytosis of spine: a comparative study of clinical, imaging features, and diagnosis in children, adolescents, and adults. Spine J 2013; 13: 1108-1117.

Jiang L, Liu XG, Zhong WQ, Ma QJ, Wei F, Yuan HS, Dang GT, Liu ZJ. Langerhans cell histiocytosis with multiple spinal nvolvement. Eur Spine J 2011; 20: 1961-1969.

Krengli M, Boccardi A, Gandini G, Negri G. Report of a rare case of Langerhans-cell histiocytosis of the skull base. Minerva Medica 1996; 87: 99.

Lazio BE and Stambough JL. An unusual posterior element spine tumor. Orthopedics 1994, 17: 725-728.

Lee SW, Kim H, Suh JK, Koh KN, Im HJ, Yoon HM, Seo JJ. Long-term clinical outcome of spinal Langerhans cell histiocytosis in children. Int J Hematol 2017; 106: 441-449.

Li Haogang, Guo Xuejun, Li Xingrong. Application value of MRI, CT and DR in the diagnosis of spinal metastasis. Chinese Journal of Clinicians 2012; 6: 1326-1328. 745

Li Ruiqi. Analysis of imaging characteristics of eosinophilic granuloma of spine. Modern medical imaging 2015; 24: 743-

Reddy PK, Vannemreddy PS, Nanda A. Eosinophilic granuloma of spine in adults: a case report and review of literature. Spinal Cord 2000; 38: 766-768.

Sweasey TA and Dauser RC. Eosinophilic granuloma of the cervicothoracic junction. J Neurosurg 1989; 71: $942-944$.

Xu Chen, Yang Xianwei, Zheng Yun. MRI image differentiation of spinal tuberculosis and non tuberculous spondylitis. Clin Radiol 2015; 34: 960-966.

Zhang Lihua and Yuan huishu. Imaging analysis and differential diagnosis of spinal LCH. Clin Radiol 2019; 5.

Zhang S, Huangfu Y, Gong L. CT and MRI diagnoxsis of Langerhans cell histiocytosis of skull. Clin Radiol 2010: 38-406.

Zhu Y, Zhou X, Meng K. Immunophenotype and electron microscopy of Langerhans cell histiocytosis. Chinese Journal of Pathology 2003; 32: 265-266. 